\title{
Case Report: Khat Chewing and Acute Myocardial Infarction in Two Young Men without Underlying Risk Factors
}

\author{
Ahmed Abdulahi Hussen, ${ }^{1,2} \dagger$ Foziya Mohammed Hussien, ${ }^{3 *} \dagger$ Nejib Yusuf, ${ }^{2}$ Aragaw Yimer Ahmed, ${ }^{4}$ and Hamid Yimam Hassen ${ }^{5}$ \\ ${ }^{1}$ Department of Internal Medicine, College of Health and Medical Sciences, Jigjiga University, Jigjiga, Ethiopia; ${ }^{2}$ Department of Internal Medicine, \\ College of Health and Medical Sciences, Haramaya University, Harer, Ethiopia; ${ }^{3}$ Department of Public Health, College of Medicine and Health \\ Sciences, Wollo University, Dessie, Ethiopia; ${ }^{4}$ Department of Internal Medicine, College of Medicine and Health Sciences, Wollo University, Dessie, \\ Ethiopia; ${ }^{5}$ Department of Public Health, College of Medicine and Health Sciences, Mizan-Tepi University, Mizan Teferi, Ethiopia 1145
}

\begin{abstract}
Khat (Catha edulis) chewing is linked to several social, psychological, and health-related problems. Studies show that khat is associated with gastrointestinal and nervous system diseases. However, little is known about khat's effect on the cardiovascular system. This case report describes acute myocardial infarction (AMI) among two young adults who chew khat frequently, but who do not have underlying cardiovascular disease (CVD) risk factors. Case 1 is a 29-year-old apparently healthy man who presented with severe, squeezing, left-side chest pain after consumption of khat. Most of the laboratory results were within the normal range except for his serum troponin level, which was 400 times more than the normal limit. The patient was diagnosed with Killip class IV, ST-segment elevation, anteroseptal AMI. Case 2 is a 25year-old man who is a frequent khat chewer. He presented with sudden-onset, severe, squeezing, retrosternal chest pain after khat chewing and vigorous activity. The patient was diagnosed with (Killip class III) acute ST-elevation myocardial infarction with cardiogenic pulmonary edema. These case reports describe two young adult male patients who were confirmed of having AMI with no known risk factors. Both cases had a similar history of frequent khat chewing and the onset of $\mathrm{AMl}$ after it, implying that khat could be an important CVD risk factor among young adults. Hence, it is essential to explore further the epidemiology and association between khat use and AMI. Both molecular and population-level studies could help to establish the causal relationship of khat and CVD.
\end{abstract}

\section{INTRODUCTION}

Khat refers to the leaves and shoots of the plant Catha edulis Forsk, which belongs to the plant family Celastraceae. ${ }^{1}$ The leaves of khat are chewed by people to reduce fatigue and enhance performance and confidence, and for its pleasurable effects, including euphoria, loquacity, and excitement. ${ }^{2-4}$ Khat contains many different compounds, including alkaloids, terpenoids, flavonoids, sterols, glycosides, tannins, amino acids, vitamins, and minerals., ${ }^{3,5}$ Alkaloids, including phenylalkylamines and cathinone, which are related structurally to amphetamine, exert an effect on the central nervous system and lead to euphoria and stimulation., ${ }^{3,5,6}$

For the past three decades, khat has become increasingly available worldwide and its use is perpetuated. ${ }^{2}$ It is common in the Horn of Africa, Arabian Peninsula, and East Coast of Africa including Ethiopia, and it has been part of the social and cultural heritage for centuries. ${ }^{2,4}$ Although, the global figure of Khat users is unknown, it is estimated to be more than 20 million. $^{7}$

Khat chewing is linked to social, psychological, and healthrelated qualities of life. ${ }^{8,9}$ The major health effects are on the gastrointestinal and nervous systems. Peripheral nervous system effects include constipation, urine retention, and acute cardiovascular effect; increased alertness, dependence, tolerance, and psychiatric symptoms are central nervous system effects. ${ }^{1}$ Similarly, the toxic effects include increased blood pressure (BP), tachycardia, insomnia, anorexia, constipation, general malaise, irritability, migraine, and impaired sexual potency in men. ${ }^{3}$

*Address correspondence to Foziya Mohammed Hussien, Department of Public Health, College of Medicine and Health Sciences, Wollo University, Wollo University Street, Dessie, Ethiopia 1145. E-mail: foziyamohammed2018@gmail.com

†These authors contributed equally to this work.
Cathinone, the main stimulant in khat, is shown to be associated with cardiovascular and neurological toxicity. ${ }^{10-12}$ It has vasoconstrictor activity that leads to high BP. ${ }^{13}$ Thus, its effect on BP might be related to the increased incidence of acute myocardial infarction (AMI) that occurs during the khat-effective period and is associated with heavy khat chewing. ${ }^{14-16}$ Alkadi et al. ${ }^{17}$ indicated that three fourths of patients with myocardial infarction have a history of khat chewing. A systematic review also indicated a higher BP and heart rate among khat chewers than their counterparts. ${ }^{18}$ Nevertheless, these studies focused on the aggravating and moderating effect of khat among older adults and among those with underlying risk factors. Our case report describes the clinical presentation, and laboratory and imaging results of two young adults diagnosed with myocardial infarction with a history of frequent khat chewing but no known underlying risk factors, which could be baseline evidence for a further large-scale, controlled analytical study.

\section{CASE 1}

A 29-year-old man with a lean body mass, previously apparently healthy, and a non-smoker with no family history of heart disease presented with severe, squeezing, left-side chest pain radiating to the neck, shoulder, and left arm of 3 days' duration. The pain was accompanied by palpitation and diaphoresis. He has been cultivating and consuming khat for a long period of time. He started feeling pain after he carried a 22-kg water pump generator immediately after chewing khat.

By late afternoon of the second day, his condition was worsening, with severe shortness of breath and fatigue. As a result, he was taken to the nearby health center, then referred to Hiwot Fana Specialized University Hospital. The patient had no personal or family history of diabetes, hypertension, or cardiac disease. Upon arrival at the emergency room, the patient was acutely sick looking, diaphoretic, exhausted, and distressed. 
On examination, the patient had tachycardia, tachypnea, hypoxia (pulse oxygen saturation, 86\%), and cardiogenic shock (BP, 100/32 mmHg) with low-grade fever (Table 1). In addition, he had intercostal and subcostal retraction using accessory muscles, and crepitation and decreased air entry over the lower bilateral lung fields, with raised jugular pressure. On pericardial examination, he had quit pericardium with muffled heart sounds, but no murmur or gallop was detected. Furthermore, there was no sign of fluid collection, organomegaly, and edema on the musculoskeletal system. He was conscious; oriented to time, place, and person; and had a Glasgow Coma Scale of 15 of 15.

Most of the baseline laboratory tests were in the normal range except for his serum troponin level, which was significantly greater and persistently more than 400 times the normal upper limit (Table 2). Electrocardiography (EKG) showed an ST-segment elevation with anteroseptal wall involvement and right bundle branch block (Figure 1).

Echocardiography showed a mildly dilated left atrium and left ventricle (LV), with normal wall thickness. Left ventricle global systolic function was severely reduced (ejection fraction, 25-30\%). The anterior, anteroseptal, anterolateral, and apical left ventricle wall were hypokinetic. Also noted was a large left ventricle apical thrombus measuring $27 \times 28 \mathrm{~mm}$. In addition, there was mild mitral regurgitation and tricuspid regurgitation, with a peak velocity of $3.2 \mathrm{~m} / \mathrm{s}$, and signs of mild pulmonary hypertension (Figure 2).

The patient was diagnosed with Killip class IV ST-segment elevation, anteroseptal AMI, and medical management were initiated accordingly. After being transferred to the medical intensive care unit (ICU), he was treated with aspirin. He was treated then with other medications, including atorvastatin, clopidogrel, heparin, morphine, tramadol, cimetidine, bisacodyl, and warfarin when needed. Similarly, he was recommended strict bed rest, and his serum troponin level was monitored. Subsequently, he became hemodynamically stable without the support of vasopressors. Within the first 2 days of admission, the patient was able to maintain oxygen saturation. On day 3 of admission, he started metoprolol after he achieved a stable BP and his chest crepitation had cleared. Despite the progressive and significant clinical improvement, his serum troponin level remained high and his EK showed no change.

After clinical follow-up, the patient was transferred to the medical ward and discharged after 7 days of observation, with an appointment for medical follow-up after 15 days. However, the patient did not return for follow-up and his progress after discharge is unknown.

TABLE 1

Vital sign results of case 1, Hiwot Fana Specialized University Hospital, Ethiopia, 2020

\begin{tabular}{lc}
\hline \multicolumn{1}{c}{ Vital sign } & Result \\
\hline Blood pressure & $100 / 32 \mathrm{mmHg}$ \\
Pulse rate & 109 beats $/ \mathrm{min}$ \\
Respiratory rate & 30 breaths $/ \mathrm{min}$ \\
Temperature & $37.6^{\circ} \mathrm{C}$ \\
Pulse oxygen saturation & $86 \%$ \\
Weight & $68 \mathrm{~kg}$ \\
Height & $172 \mathrm{~cm}$ \\
Body mass index & $23 \mathrm{~kg} / \mathrm{m}^{2}$ \\
\hline
\end{tabular}

\section{CASE 2}

A 25-year-old male khat farmer and chewer, previously healthy and a non-smoker, presented with sudden-onset, severe, squeezing retrosternal chest pain of 6 hours' duration, and tightness radiating to the left, right, and back of the chest. The pain started after vigorous work after chewing khat for more than an hour in the afternoon. The patient described it as "the worst pain of my life." Subsequently, the patient collapsed and was taken to a nearby health center by ambulance. He was later referred to Hiwot Fana Specialized University Hospital. The patient had no known personal or family history of diabetes, hypertension, or cardiovascular or renal diseases.

On examination, the patient was acutely sick looking and in respiratory distress. He also had tachypnea and tachycardia, and was severely hypoxic (pulse oxygen saturation, 76\%) and agitated (Table 3). Furthermore, he had bilateral diffuse crepitation, decreased air entry in the bilateral lower third of the lung, distended neck veins, quit pericardium with S3 gallop, and tender hepatomegaly with no sign of fluid collection. He was oriented consciously to time, place, and person. In addition, the patient had pink conjunctiva, non-icteric sclera, and white buccal mucosa.

The majority of his laboratory results were within the normal range, but his serum troponin level was 400 times greater than the normal limit. Chest X-ray showed bilateral pleural effusion with marked pulmonary edema (Table 4). EKG revealed anterolateral acute ST-elevation myocardial infarction with sinus tachycardia (Figure 3).

Echocardiography showed global systolic dysfunction of the left ventricle, with a severely reduced ejection fraction estimated at $20 \%$. There was motion abnormality in the anterior, anteroseptal, and anterolateral walls of the left ventricle (Figure 4).

The patient was diagnosed with Killip class III ST-elevation myocardial infarction with cardiogenic pulmonary edema. Subsequently, he was seated under intranasal oxygen and monitored with EKG, and later admitted to the medical intensive care unit, where he was administered Lasix. In addition, his BP and urine output were monitored intensely, and he was administered aspirin, atorvastatin, clopidogrel, heparin, morphine, tramadol, cimetidine, bisacodyl, and warfarin as needed. After 5 days in the medical ICU, with clinical improvement and complete resolution of symptoms, the patient was transferred to the medical inpatient unit. Subsequently, he disappeared from the hospital without informing physicians and his further progress is unknown.

\section{DISCUSSION}

In 2019, with an estimated 523 million cases and 18.6 million deaths, cardiovascular diseases (CVDs) are the leading cause of adult morbidity and mortality worldwide. ${ }^{19}$ Among CVDs, ischemic heart disease and stroke are the first and second leading causes of death respectively. ${ }^{19,20}$ In the past three decades, a decline in the age-standardized CVD deaths has been observed in high-income countries; however, the burden in most low- and middle-income countries has increased, contributing to three quarters of all global CVD deaths. ${ }^{21} \mathrm{Sim}-$ ilarly, in Ethiopia, with an overall death rate of 182.6 per 100,000 population, CVDs are the leading cause of 
TABLE 2

Laboratory investigation results of case 1, Hiwot Fana Specialized University Hospital, Ethiopia, 2020

\begin{tabular}{|c|c|c|c|c|c|}
\hline \multirow[b]{2}{*}{ Laboratory test } & \multicolumn{5}{|c|}{ Laboratory results } \\
\hline & Day 1 & Day 2 & Day 4 & Day 7 & Normal range \\
\hline White blood cell (cells $/ \mu \mathrm{L}$ ) & 14,100 & - & - & - & $4,000-11,000$ \\
\hline Neutrophil (\%) & 74.8 & - & - & _- & $55-75$ \\
\hline Lymphocyte (\%) & 17.8 & - & - & - & $25-40$ \\
\hline Hemoglobin $(\mathrm{g} / \mathrm{dL})$ & 12.8 & - & - & - & $12-16$ \\
\hline Hematocrit (\%) & 38.3 & - & - & - & $36-46$ \\
\hline Platelet (cells $/ \mu \mathrm{L})$ & 303,000 & - & - & - & $150,000-450,000$ \\
\hline Random blood sugar (mg/dL) & 139 & - & - & - & $<200$ \\
\hline Creatinine $(\mathrm{mg} / \mathrm{dL})$ & 0.9 & - & 0.7 & - & $0.5-1.2$ \\
\hline Blood urea nitrogen $(\mathrm{mg} / \mathrm{dL})$ & 62 & - & - & - & $13-43$ \\
\hline Serum glutamic oxaloacetic transaminase (U/L) & 81 & - & - & - & $12-38$ \\
\hline Serum glutamic pyruvic transaminase $(\mathrm{U} / \mathrm{L})$ & 93 & - & - & - & $7-41$ \\
\hline Alkaline phosphatase level (U/L) & 141 & - & - & - & $60-306$ \\
\hline Bilirubin total (mg/dL) & 0.8 & - & - & - & $<1.2$ \\
\hline Direct bilirubin (mg/dL) & 0.7 & - & - & - & $<0.4$ \\
\hline Serum $\mathrm{K}^{+}(\mathrm{mmol} / \mathrm{L})$ & 3.71 & _- & _- & 3.9 & $3.5-5.0$ \\
\hline Serum $\mathrm{Na}^{+2}(\mathrm{mmol} / \mathrm{L})$ & 141.3 & - & 143 & - & $136-146$ \\
\hline Chlorine (mmol/L) & 106.2 & - & 109.5 & - & $102-109$ \\
\hline Serum ionized $\mathrm{Ca}(1.2$ to $1.4 \mathrm{~L})$ & 1.3 & - & - & - & \\
\hline Serum troponin $(\mathrm{ng} / \mathrm{L})$ & $>40,000$ & $>40,000$ & $>40,000$ & $>40,000$ & $\begin{array}{c}<2 \text {, rule out; } 1-100 \\
\text { observe; }>100, \text { rule } \\
\text { in }\end{array}$ \\
\hline Activated partial thromboplastin time (seconds) & 24.59 & - & - & - & $25-43$ \\
\hline Prothrombin time (seconds) & 16.27 & - & - & - & $13-15$ \\
\hline International normalized ratio & 1.18 & - & - & - & $0.8-1.2$ \\
\hline Chest X-ray & Right-side pleural effusion & - & - & - & - \\
\hline Abdominal ultrasound & Normal & - & - & - & - \\
\hline
\end{tabular}

mortality. ${ }^{22}$ Hence, besides interventions on the known lifestyle and metabolic risk factors, it is crucial to identify context-specific risk factors to halt the growing burden of CVDs.
AMI is an event of myocardial cell death caused by a prolonged, unstable ischemic syndrome. ${ }^{23}$ Myocardial infarction is considered acute if there is a rise and/or fall of serum troponin levels. ${ }^{23} \mathrm{AMI}$ is a common disease, with serious

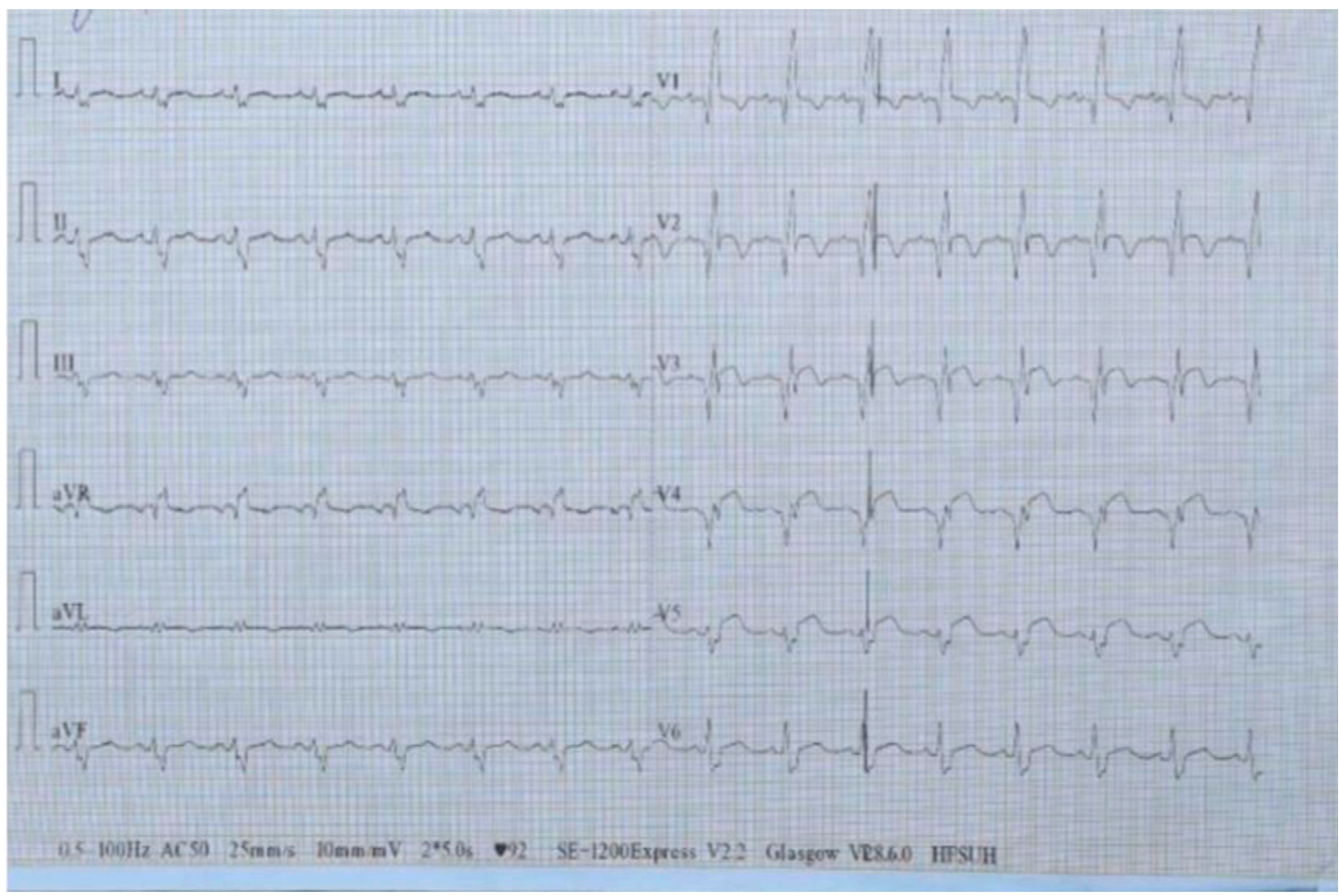

FIGURE 1. Electrocardiography results of case 1 on presentation at Hiwot Fana Specialized University Hospital, Ethiopia, 2020. 


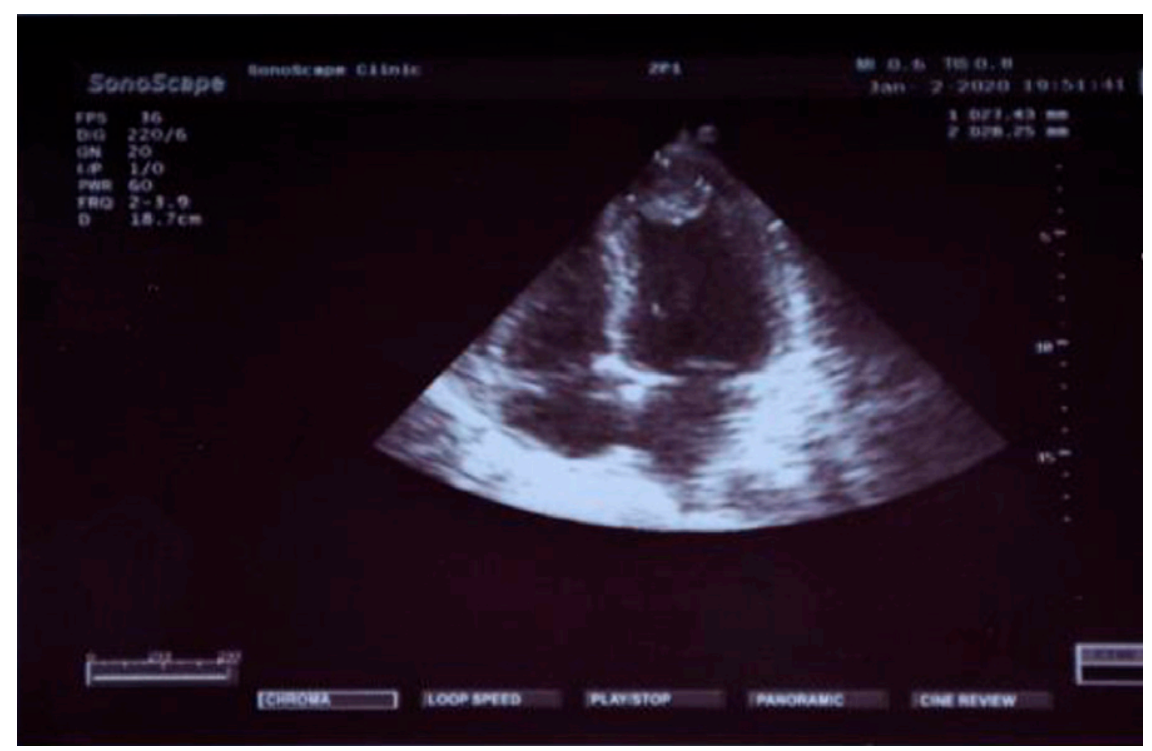

FIGURE 2. Echocardiography results of case 1, Hiwot Fana Specialized University Hospital, Ethiopia, 2020.

consequences to the community, including mortality, morbidity, and economic burden. ${ }^{24}$ It remains a leading cause of morbidity and mortality worldwide. ${ }^{25}$ AMl can be prevented if effective intervention strategies target the risk factors. ${ }^{26}$ Therefore, studies from all levels are important to identify risk factors as well as to design and implement contextspecific interventions.

Khat is widespread in Ethiopia, particularly in the eastern and southwestern regions, where its cultivation predominantly takes place. ${ }^{27,28}$ Accordingly, the number of khat chewers has been increasing rapidly in recent years, and khat chewing is gaining popularity in all segments of the community. The overall prevalence of khat chewing in Ethiopia is estimated to be $15.3 \% .^{28}$

Because khat is mainly cultivated and chewed in the Horn of Africa and the Arabian Peninsula, it has not gained the global attention of health scientists. These countries have limited resource and research infrastructures to study the effect of khat at the molecular and population level. As a result, international non-governmental organizations and civic societies do not consider it to be an important public health issue. Some studies have shown that khat chewing is associated with several somatic health sequelae, including systemic hypertension, liver diseases, upper gastrointestinal cancers, cognitive impairment, and impaired fetal growth. ${ }^{29-31}$ A systematic review by Mega and Dabe ${ }^{18}$ showed that khat is associated with an increase in BP and heart rate. Recently, a few studies

TABLE 3

Vital sign results of case 2, Hiwot Fana Specialized University Hospital, Ethiopia, 2020

\begin{tabular}{lc}
\hline \multicolumn{1}{c}{ Vital sign } & Result \\
\hline Blood pressure & $110 / 70 \mathrm{mmHg}$ \\
Pulse rate & 110 beats $/ \mathrm{min}$ \\
Respiratory rate & 30 breaths $/ \mathrm{min}$ \\
Temperature & $36.8^{\circ} \mathrm{C}$ \\
Pulse oxygen saturation & $76 \%$ \\
Weight & $72 \mathrm{~kg}$ \\
Height & $170 \mathrm{~cm}$ \\
Body mass index & $25.1 \mathrm{~kg} / \mathrm{m}^{2}$ \\
\hline
\end{tabular}

have suggested the aggravating effect of khat chewing on CVDs and risks including myocardial infarction. ${ }^{31,32}$ Such effects could be the result of cathinone toxicity, which is the main stimulant in khat.

Both patients in our case report have been consuming khat daily for a long period of time. They had no known risk factors for AMI, such as smoking, family and personal histories of cardiac diseases, and other comorbid illnesses. Moreover, the acute pain they experienced occurred after chewing khat. Therefore, in these patients, khat chewing might be the independent risk factor of $\mathrm{AMI}$, despite the fact that we cannot conclude this without a controlled group and adjusting for other covariates. This finding is consistent with several studies in different parts of the world that reported that many patients presented with AMI in the evening either during or after a khatchewing session. ${ }^{14-16,33-35}$ Increases in BP and heart rate were observed in khat chewers, which coincide with raised plasma cathinone concentrations leading to AMI, similar to amphetamine. ${ }^{13}$ Therefore, the effect of khat on CVDs-and

TABLE 4

Laboratory investigation results of case 2, Hiwot Fana Specialized University Hospital, Ethiopia, 2020

\begin{tabular}{|c|c|c|}
\hline Test & Result & Normal range \\
\hline $\begin{array}{l}\text { White blood cell } \\
\text { (cells } / \mu \mathrm{L})\end{array}$ & 3,277 & $4,000-11,000$ \\
\hline Neutrophil (\%) & 94 & $55-75$ \\
\hline Lymphocyte (\%) & 3 & $25-40$ \\
\hline Hemoglobin (mg/dL) & 14 & $12-16$ \\
\hline Hematocrit (\%) & 45.8 & $36-46$ \\
\hline Platelet (cells/ $\mu \mathrm{L})$ & 268,000 & $150,000-450,000$ \\
\hline $\begin{array}{l}\text { Random blood sugar } \\
(\mathrm{mg} / \mathrm{dL})\end{array}$ & 175 & $<200$ \\
\hline Troponin (ng/L) & $>40,000$ & $\begin{array}{l}<2, \text { rule out; } 1-100 \\
\text { observe; }>100, \text { rule in }\end{array}$ \\
\hline $\mathrm{Na}^{+2}(\mathrm{mmol} / \mathrm{L})$ & 139.6 & $136-146$ \\
\hline $\mathrm{K}^{+}(\mathrm{mmol} / \mathrm{L})$ & 4.66 & $3.5-5.0$ \\
\hline $\mathrm{Cl}^{-}(\mathrm{mmol} / \mathrm{L})$ & 103 & $102-109$ \\
\hline Chest X-ray & $\begin{array}{l}\text { Bilateral pleural } \\
\text { effusion with feature } \\
\text { of pulmonary } \\
\text { edema }\end{array}$ & - \\
\hline
\end{tabular}




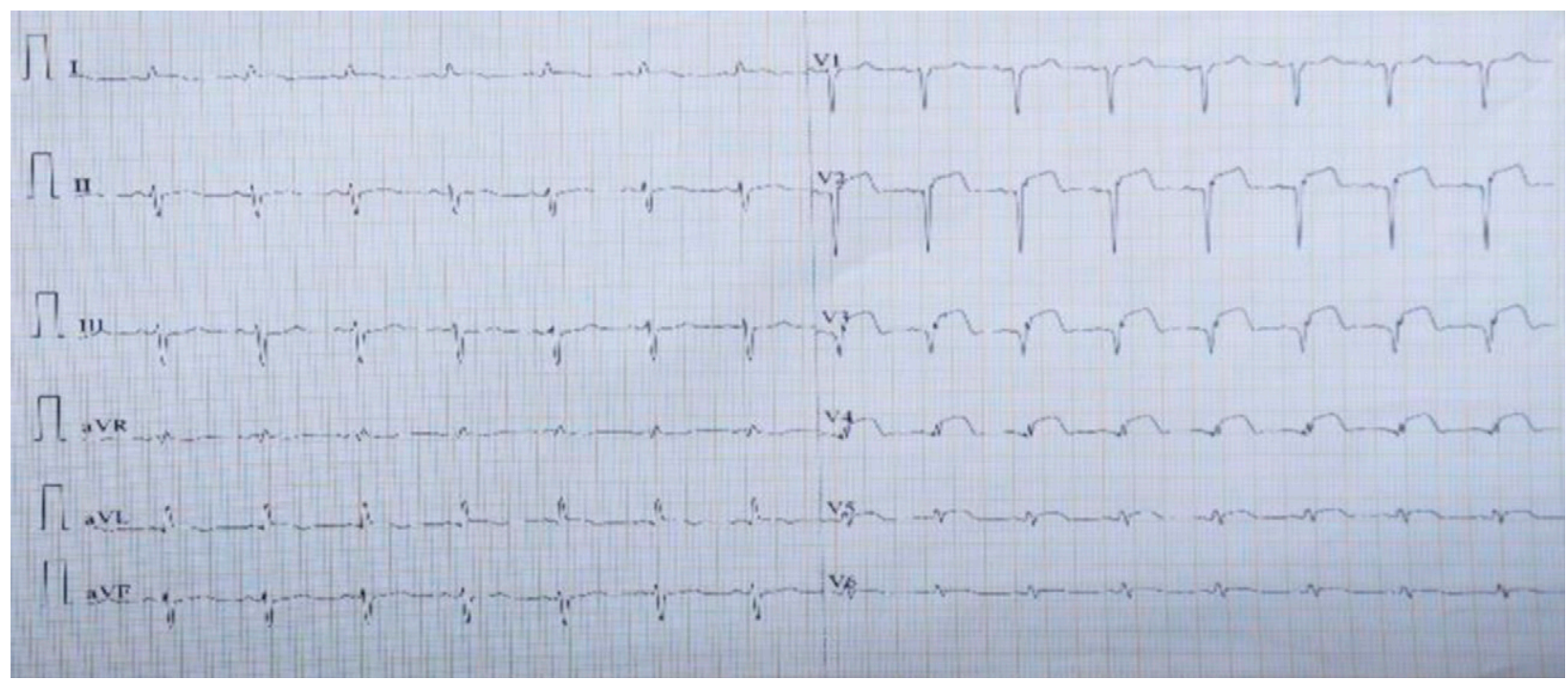

FIGURE 3. Electrocardiography results of case 2, Hiwot Fana Specialized University Hospital, Ethiopia, 2020.

AMI in particular-needs to be studied using controlled groups and a larger sample. Large-scale prospective studies with long-term follow-up could help to establish the causal relationship of khat on CVDs and other organ systems. In the meantime, health education for high-risk individuals such as khat farmers and merchants could help minimize the risk of developing AMI. In addition, in collaboration with other sectors, public health practitioners need to design and implement intervention strategies to decrease the rate of khat chewing.

Practically, AMI is diagnosed and assessed based on clinical evaluation, including EKG, biochemical testing, invasive and noninvasive imaging, and pathological evaluation. ${ }^{24} \mathrm{Sim}-$ ilarly, our patients were evaluated with the combined diagnostic modalities. Unfortunately, their financial situation negated further investigation, such as acquiring a lipid profile and repeated measurements, and monitoring their progress. Because these patients did not return for follow-up, we were unable to ascertain their outcome.
In low- and middle-income countries, including Ethiopia, medical treatment is routinely substandard, similar to the management of the presented cases. In settings with a better infrastructure, advanced imaging (cardiac perfusion imaging) and advanced management (percutaneous coronary intervention) could have been done, which is only available at the central level (Black Lion Hospital) in Ethiopia.

\section{CONCLUSION}

In general, our case reports indicate that the two young adult men were confirmed to have AMl with no known underlying risk factors. Both cases had a similar history of frequent khat chewing and the onset of AMI immediately thereafter. This shows that khat chewing could be an important risk factor for AMI in young adults. Hence, it is essential to explore the epidemiology and causal relationships further. Moreover, molecular studies could help to investigate the pathophysiological mechanism of khat on AMI and other CVDs.

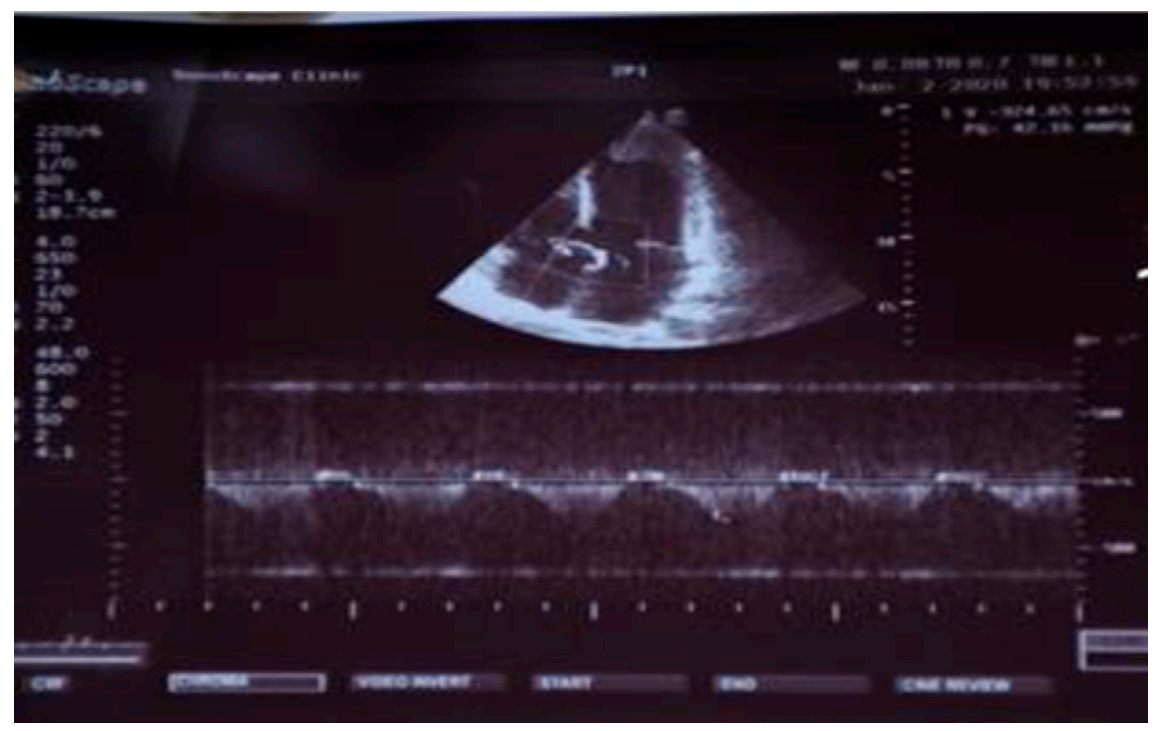

FIGURE 4. Echocardiography results of case 2, Hiwot Fana Specialized University Hospital, Ethiopia, 2020. 
Received March 18, 2021. Accepted for publication May 31, 2021.

Published online July 26, 2021.

Acknowledgment: The American Society of Tropical Medicine and Hygiene (ASTMH) assisted with publication expenses.

Authors' addresses: Ahmed Abdulahi Hussen, Department of Internal Medicine, College of Health and Medical Sciences, Jigjiga University, Jigjiga, Ethiopia, and Department of Internal Medicine, College of Health and Medical Sciences, Haramaya University, Harer, Ethiopia, E-mail: ahmediya101@gmail.com. Foziya Mohammed Hussien, Department of Public Health, College of Medicine and Health Sciences, Wollo University, Dessie, Ethiopia, E-mail: foziyamohammed2018@gmail.com. Nejib Yusuf, Department of Internal Medicine, College of Health and Medical Sciences, Haramaya University, Harer, Ethiopia, E-mail: nejibyusuf@yahoo. com. Aragaw Yimer Ahmed, Department of Internal Medicine, College of Medicine and Health Sciences, Wollo University, Dessie, Ethiopia, E-mail: arades2002@gmail.com. Hamid Yimam Hassen, Department of Public Health, College of Medicine and Health Sciences, Mizan-Tepi University, Mizan Teferi, Ethiopia, E-mail: abdulhamidy71@gmail.com.

\section{REFERENCES}

1. World Health Organization Expert Committee on Drug Dependence, 2006. Assessment of khat (Catha edulis Forsk). Proceedings of the 34th Meeting, Expert Committee on Drug Dependence. Geneva, Switzerland: WHO.

2. Krikorian AD, 1984. Khat and its use: an historical perspective. J Ethnopharmacol 12: 115-178.

3. Wabe NT, 2011. Chemistry, pharmacology, and toxicology of khat (Catha edulis Forsk): a review. Addict Health 3: 137.

4. Patel NB, 2019. Khat (Catha edulis Forsk): and now there are three. Brain Res Bull 145: 92-96.

5. Toennes SW et al., 2003. Pharmacokinetics of cathinone, cathine and norephedrine after the chewing of khat leaves. $\mathrm{Br} \mathrm{J}$ Clin Pharmacol 56: 125-130.

6. Feyissa AM, Kelly JP, 2008. A review of the neuropharmacological properties of khat. Prog Neuropsychopharmacol Biol Psychiatry 32: $1147-1166$.

7. Patel NB, 2015. "Natural amphetamine" khat: a cultural tradition or a drug of abuse? International Review of Neurobiology. Netherlands: Elsevier, 235-255.

8. Gebrie A et al., 2018. Prevalence and predictors of khat chewing among Ethiopian university students: a systematic review and meta-analysis. PLoS One 13: e0195718.

9. Sheikh KA et al., 2014. Khat chewing and health-related quality of life: cross-sectional study in Jazan region, Kingdom of Saudi Arabia. Health Qual Life Outcomes 12: 44.

10. Bentur Y, Bloom-Krasik A, Raikhlin-Eisenkraft B, 2008. Illicit cathinone ("Hagigat") poisoning. Clin Toxicol 46: 206-210.

11. Capriola M, 2013. Synthetic cathinone abuse. Clin Pharmacol 5: 109.

12. Prosser JM, Nelson LS, 2012. The toxicology of bath salts: a review of synthetic cathinones. J Med Toxicol 8: 33-42.

13. Brenneisen R et al., 1990. Amphetamine-like effects in humans of the khat alkaloid cathinone. Br J Clin Pharmacol 30: 825-828.

14. Al-Motarreb A et al., 2002. Khat chewing and acute myocardial infarction. Heart 87: 279-280.
15. Al-Motarreb A et al., 2005. Khat chewing is a risk factor for acute myocardial infarction: a case-control study. Br J Clin Pharmacol 59: 574-581.

16. Al-Motarreb A, Al-Habori M, Broadley KJ, 2010. Khat chewing, cardiovascular diseases and other internal medical problems: the current situation and directions for future research. J Ethnopharmacol 132: 540-548.

17. Alkadi $\mathrm{HO}$ et al., 2002. Clinical and experimental evaluation of the effect of khat-induced myocardial infarction. Saudi Med J 23: 1195-1198.

18. Mega TA, Dabe NE, 2017. Khat (Catha edulis) as a risk factor for cardiovascular disorders: systematic review and meta-analysis. Open Cardiovasc Med J 11: 146.

19. Roth GA et al., 2020. Global burden of cardiovascular diseases and risk factors, 1990-2019: update from the GBD 2019 study. J Am Coll Cardiol 76: 2982-3021.

20. World Health Organization, 2017. Cardiovascular Diseases (CVDs). Available at: https://www.who.int/news-room/factsheets/detail/cardiovascular-diseases-(cvds). Accessed June 11, 2021.

21. Roth GA et al., 2017. Global, regional, and national burden of cardiovascular diseases for 10 causes, 1990 to 2015. J Am Coll Cardiol 70: 1-25.

22. Ali $\mathrm{S}$ et al., 2020. The burden of cardiovascular diseases in Ethiopia from 1990 to 2017: evidence from the Global Burden of Disease Study. Int Health.

23. Thygesen $\mathrm{K}$ et al., 2018. Fourth universal definition of myocardial infarction (2018). J Am Coll Cardiol 72: 2231-2264.

24. Anderson JL, Morrow DA, 2017. Acute myocardial infarction. $N$ Engl J Med 376: 2053-2064.

25. Reed GW, Rossi JE, Cannon CP, 2017. Acute myocardial infarction. Lancet 389: 197-210.

26. Jamil $G$ et al., 2013. Risk factor assessment of young patients with acute myocardial infarction. Am J Cardiovasc Dis 3: 170.

27. Gebissa E, 2010. Taking the place of food: khat in Ethiopia.Trenton, NJ: AfricaBib, The Red Sea Press, 46: 239.

28. Haile D, Lakew Y, 2015. Khat chewing practice and associated factors among adults in Ethiopia: further analysis using the 2011 demographic and health survey. PLoS One 10: e0130460.

29. Corkery JM et al., 2011. Overview of literature and information on "khat-related" mortality: a call for recognition of the issue and further research. Ann Ist Super Sanita 47: 445-464.

30. Mahamoud HD et al., 2016. Khat chewing and cirrhosis in Somaliland: case series. Afr J Prim Health Care Fam Med 8: 1124.

31. Nigussie T, Gobena T, Mossie A, 2013. Association between khat chewing and gastrointestinal disorders: a cross sectional study. Ethiop J Health Sci 23: 123-130.

32. Aronson JK, ed., 2016. Celastraceae. Meyler's Side Effects of Drugs, 16th edition. Oxford, UK: Elsevier, 184-190.

33. Al-Motarreb A, Baker K, Broadley KJ, 2002. Khat: pharmacological and medical aspects and its social use in Yemen. Phytother Res 16: 403-413.

34. Ali WM et al., 2010. Association of khat chewing with increased risk of stroke and death in patients presenting with acute coronary syndrome. Mayo Clinic Proceedings 85: 974-980. Netherlands: Elsevier.

35. Saha S, Dollery C, 2006. Severe ischaemic cardiomyopathy associated with khat chewing. J R Soc Med 99: 316-318. 